\title{
氯化镁矿化利用低浓度烟气 $\mathrm{CO}_{2}$ 联产碳酸镁
}

谢和平 ${ }^{(12) *}$ ，王昱飞 ${ }^{(1)(2)}$ ，储伟 ${ }^{(1)}$ ，鞄杨 ${ }^{(1)}$

(1) 四川大学 CCUS 与 $\mathrm{CO}_{2}$ 矿化利用研究中心, 成都 610065;

(2) 四川大学水利水电学院, 能源工程安全与灾害力学教育部重点实验室, 成都 610065;

(3) 四川大学化学工程学院, 成都 610065 ;

(4.) 中国矿业大学, 煤炭资源与安全开采国家重点实验室, 北京 100083

* 联系人, E-mail: xiehp@scu.edu.cn

2014-02-03 收稿, 2014-04-09 接受, 2014-05-04 网络版发表

国家杰出青年科学基金(51125017)、科技部国际科技合作项目(2012DFA60760)、国家自然科学基金(51120145001, 51254002)和国家重点基 础研究发展计划(2011CB201201, 2010CB226804)资助

摘要 $\mathrm{CO}_{2}$ 矿化利用是一种新的 $\mathrm{CO}_{2}$ 减排方式, 主要原理是利用天然矿物或工业废料与 $\mathrm{CO}_{2}$ 反应, 将 $\mathrm{CO}_{2}$ 矿化为碳酸钙或碳酸镁等固体碳酸盐, 同时联产高附加值的化工产品。本文提出 采用低能耗的电解工艺利用氯化镁溶液矿化利用低浓度烟气 $\mathrm{CO}_{2}$ 的新方法, 该方法将氯化镁 转化为活性较高的氢氧化镁, 与不同浓度的 $\mathrm{CO}_{2}$ 反应从而矿化 $\mathrm{CO}_{2}$. 该方法可以直接矿化浓 度仅为 $20 \%$ 的低浓度 $\mathrm{CO}_{2}$, 这使得直接矿化利用工业烟气 $\mathrm{CO}_{2}$ 成为可能, 避免了较高能耗的 $\mathrm{CO}_{2}$ 捕捉提纯过程. 另外, 该方法可在较低的能耗下减排大量 $\mathrm{CO}_{2}$, 同时获取高附加值的碱式 碳酸镁或三水合碳酸镁. 由于氯化镁自然储量非常丰富, 氯化镁矿化利用 $\mathrm{CO}_{2}$ 具有大规模减 排的潜力.

化石燃料燃烧排放的 $\mathrm{CO}_{2}$ 被广泛认为是造成 全球变暖的主要原因和人类生存环境的威胁, 减 排 $\mathrm{CO}_{2}$ 成为全球的共同目标 ${ }^{[1 \sim 5]}$. 对 $\mathrm{CO}_{2}$ 进行捕集和 封存 (CCS) 是目前主要研究的 $\mathrm{CO}_{2}$ 减排方法. $\mathrm{CCS}$ 技术首先将烟气或其他工业过程中排放的烟气 $\mathrm{CO}_{2}$ (其浓度为 $10 \%$ 20\%)进行捕捉提纯，然后再将提 纯后的 $\mathrm{CO}_{2}$ 进行封存. 目前, 烟气 $\mathrm{CO}_{2}$ 的捕捉提纯主 要采用化学吸收的方法, 其能耗较高, 从烟气中捕捉 每吨 $\mathrm{CO}_{2}$ 的能耗为 $370 \sim 510 \mathrm{~kW} \mathrm{h.} \mathrm{CO}_{2}$ 地质封存是目 前研究最多的 $\mathrm{CO}_{2}$ 封存方法, 在我国鄂尔多斯以及美 国德克萨斯等地都开展了 $\mathrm{CO}_{2}$ 地质封存的现场实 验 ${ }^{[6 ~ 9]}$. 但由于 $\mathrm{CO}_{2}$ 地质封存投人较大, 并且其安全 性仍然存在争议, 因此 $\mathrm{CO}_{2}$ 地质封存技术的推广较为 缓慢 ${ }^{[10]}$.

$\mathrm{CO}_{2}$ 矿化利用是近年来新提出的一种 $\mathrm{CO}_{2}$ 封存 和利用途径, 主要利用天然矿物或工业废料与 $\mathrm{CO}_{2}$
反应, 将 $\mathrm{CO}_{2}$ 封存为碳酸钲或碳酸镁等固体碳酸盐, 同时联产高附加值的化工产品 ${ }^{[1113]}$.

目前, 矿化 $\mathrm{CO}_{2}$ 的原料主要集中于橄榄石、蛇纹 石等天然碱性硅酸盐 ${ }^{[14 ~ 18]}$. 天然硅酸盐与 $\mathrm{CO}_{2}$ 的矿 化反应在自然界中便可进行, 但这一过程过于缓慢, 不适合在短期内大规模减排 $\mathrm{CO}_{2}$. 研究表明, 通过在 反应前对天然硅酸盐进行炷烧、球磨等预活化, 同时 提高反应时的温度和 $\mathrm{CO}_{2}$ 分压, 可在短时间内高效 矿化 $\mathrm{CO}_{2}{ }^{[19-21]}$. 但这一过程不但能耗很高 ${ }^{[22]}$, 且产 物主要为碳酸盐和二氧化硅的混合物, 产品附加值 较低.

碱性工业废料是 $\mathrm{CO}_{2}$ 矿化的另一种原料 ${ }^{[23 ~ 26]}$. 能够用于矿化 $\mathrm{CO}_{2}$ 的碱性废料主要包括水泥窑粉尘、 粉煤灰以及其他富含氧化钙的生活或工业垃圾. 虽 然碱性工业废料的反应活性比硅酸盐类矿物高很多, 更容易与 $\mathrm{CO}_{2}$ 发生矿化反应, 但碱性工业废料的来 
源并不稳定, 能够矿化 $\mathrm{CO}_{2}$ 的总量有限. 另外, 利用 碱性工业废料矿化 $\mathrm{CO}_{2}$ 后的产物, 其化学成分较为 复杂, 纯度不高.

氯化镁是一种广泛分布的化合物, 主要以溶液 形式存在于海水、盐湖之中, 其储量相当丰富, 以海 水中的氯化镁为例, 海水中的平均镁含量约为 $0.13 \%$, 主要以氯化镁的形式存在. 2010 年全球 $\mathrm{CO}_{2}$ 排放量约 为 300.6 亿吨 ${ }^{[27]}$, 如果能够将海水中氯化镁的 $0.1 \%$ 用于矿化 $\mathrm{CO}_{2}$ 生成碳酸镁, 便可减排 $\mathrm{CO}_{2} 3.34$ 万亿 吨, 相当于 111 年世界 $\mathrm{CO}_{2}$ 的排放总量. 我国的氯化 镁存量相当丰富, 我国海域周围海水中的镁含量达 到 $0.13 \%$, 我国 4 大盐湖区镁盐矿产资源的远景储量 达数十亿吨. 其中, 柴达木盆地内大小不等的 33 个 卤水湖、半干涸盐湖和干涸盐湖, 蕴藏着储量占全国 第一位的镁盐资源. 另外, 利用氯化镁能够矿化利用 $\mathrm{CO}_{2}$, 可以产出具有高附加值的碳酸镁:

$$
\begin{aligned}
& 5 \mathrm{MgCl}_{2}+4 \mathrm{CO}_{2}+10 \mathrm{H}_{2} \mathrm{O} \rightarrow \\
& \mathrm{Mg}_{5}\left(\mathrm{CO}_{3}\right)_{4}(\mathrm{OH})_{2} \cdot 4 \mathrm{H}_{2} \mathrm{O}+10 \mathrm{HCl}
\end{aligned}
$$

碳酸镁不仅可用作绝热、耐高温的防火保温材料, 还可用于制造高级玻璃制品、镁盐、颜料、油漆和日 用化学品. 因此, 氯化镁具有大规模矿化利用 $\mathrm{CO}_{2}$, 联产高附加值化工产品的潜力.

然而到目前为止, 还没有直接利用氯化镁溶液矿 化利用 $\mathrm{CO}_{2}$ 的研究报道. 因此, 如何利用氯化镁溶液矿 化利用 $\mathrm{CO}_{2}$, 同时联产高附加值的碳酸镁是本文的主要 目的. 另外, 由于从烟气中捕捉 $\mathrm{CO}_{2}$ 的能耗较高, 因此, 本文的另一个目的在于探索一种直接矿化利用低浓度 烟气 $\mathrm{CO}_{2}$ 的新方法, 从而降低 $\mathrm{CO}_{2}$ 矿化利用能耗.

本文提出了一种利用氯化镁溶液矿化利用烟气 $\mathrm{CO}_{2}$ 的新方法. 该方法旨在低能耗下, 利用氯化镁溶 液矿化利用低浓度 $\mathrm{CO}_{2}$, 联产高附加值的碳酸镁, 其 工艺过程为: 通过电化学方法, 在低电压下将氯化镁 溶液转化为具有较高反应活性的氢氧化镁, 并产出 盐酸. 生成的碱性氢氧化镁易于与具有酸性的 $\mathrm{CO}_{2}$ 气体反应, 将其转化为碳酸氢镁溶液. 碳酸氢镁溶液 在一定温度下分解便可获取高附加值的碳酸镁(包括 碱式碳酸镁), 过程如图 1 所示.

低电压电解氯化镁溶液制取氢氧化镁的示意图 如图 2 所示, 阴离子交换膜将电解槽分为两部分, 并 采用氢气气体扩散电极作为正极. 氢气气体扩散电 极最早起源于燃料电池, 由钛网集流层, 碳纸和催化 层构成, 可在低电压下将氢气氧化为氢离子 ${ }^{[28 ~ 30]}$.

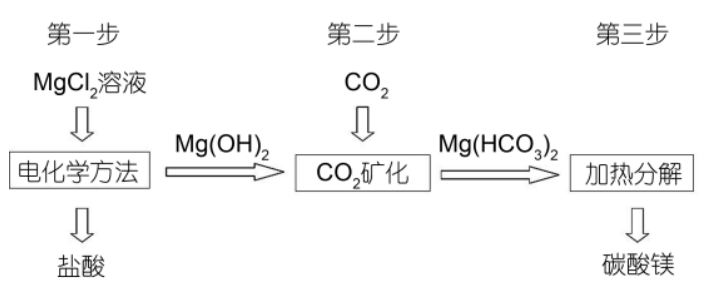

图 1 氯化镁溶液矿化利用 $\mathrm{CO}_{2}$ 示意图

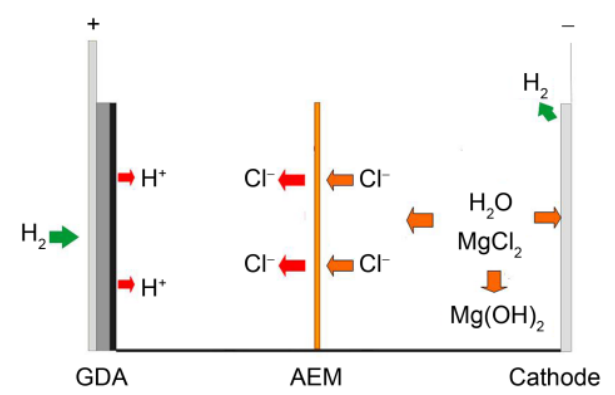

图 2 低电压电解氯化镁溶液制取氢氧化镁示意图

在电解作用下, 负电极表面产生氢气, 大量氢氧根在 负极电解液中产生, 与镁离子反应, 生成氢氧化镁沉 淀. 气体扩散电极则不断将氢气氧化成氢离子, 与从 阴离子交换膜透过的氯离子生成盐酸溶液.

负极: $2 \mathrm{H}_{2} \mathrm{O}+\mathrm{Mg}^{2+}+2 \mathrm{e}^{-} \rightarrow \mathrm{Mg}(\mathrm{OH})_{2}+\mathrm{H}_{2}$

正极: $\mathrm{H}_{2} \rightarrow 2 \mathrm{H}^{+}+2 \mathrm{e}^{-}$

本文通过以上方法, 研究了利用氯化镁溶液矿 化利用 $\mathrm{CO}_{2}$, 联产碳酸镁的可行性. 并探索了直接采 用低浓度烟气 $\mathrm{CO}_{2}$ 作为矿化原料的可能性. 对影响 氯化镁矿化利用 $\mathrm{CO}_{2}$ 过程中的关键因素, 如槽电压, 盐酸浓度, 氯化镁浓度, $\mathrm{CO}_{2}$ 分压, 分解温度等的作 用进行了研究.

\section{1 实验概况}

\section{1 实验原料}

分析纯的氯化镁、氯化钠以及盐酸被直接用作实 验原料. 纯度为 $99.99 \%$ 的 $\mathrm{CO}_{2}, \mathrm{~N}_{2}$ 和 $\mathrm{H}_{2}$ 由气体钢瓶 提供, 均购买自四川东风气体公司. 氢气气体扩散电 极购买自中国河森公司, 采用 40 目钛网作为集流层, 催化剂层是担载 $1 \mathrm{mg} \mathrm{cm}^{-2}$ 的铂炭. 阴离子交换膜购 买自中国 Iontech 公司.

\section{2 实验过程}

（i ）电解氯化镁溶液. 实验采用不同浓度的 $\mathrm{HCl}$ 溶液 $(0 \sim 1.6 \mathrm{~mol} / \mathrm{L})$ 作为正极电极液, 氯化美溶液 
$(1 \mathrm{~mol} / \mathrm{L})$ 作为负极电极液. 正极电极液中的盐酸浓 度通过酸碱滴定进行测定. 负极电极液经分离、过 滤、洗涤、干燥 $\left(110^{\circ} \mathrm{C}\right)$, 得到氢氧化镁.

采用不同浓度的盐酸作为正极电极液, 测定并 记录在不同起始浓度的盐酸正极电极液条件下，电 流密度随电压变化. 从海水或盐湖中得到的氯化镁 溶液常含有大量的氯化钠，实验过程中，不同浓度的 氯化钠和氯化镁的混合溶液被用作负极电极液, 测 定记录和分析上述几种配比条件下的电流密度随槽 电压的变化曲线.

(ii) 矿化不同浓度的 $\mathrm{CO}_{2}$ 生成碳酸氢镁. 将电 解得到的氢氧化镁置于 $250 \mathrm{~mL}$ 三口烧瓶中, 加人 $100 \mathrm{~mL}$ 蒸馏水搅拌分散, 将纯度为 $99.99 \% \mathrm{CO}_{2}$ 和 $\mathrm{N}_{2}$ 通过气体质量流量计调节流量后混合, 形成不同浓 度的 $\mathrm{CO}_{2}$ 气体, 通人上述悬浊液中, 与氢氧化镁发生 反应. 矿化反应在常温下进行, 摚拌速率为 350 $\mathrm{r} / \mathrm{min}$. 通过调节 $\mathrm{CO}_{2}$ 和 $\mathrm{N}_{2}$ 的流量, 控制通人气体的 $\mathrm{CO}_{2}$ 浓度为 $20 \% \sim 100 \%$. 反应过程中, 每隔 $15 \sim 30$ $\min$ 利用针管注射器抽出 $1 \mathrm{~mL}$ 溶液, 经针管过滤头 过滤后, 进行酸碱滴定, 测定溶液中碳酸氢根的含 量. 当溶液中的碳酸氢根含量趋于稳定后停止反应.

(iii) 分解碳酸氢镁溶液制备碳酸镁矿化产物. 将矿化 $\mathrm{CO}_{2}$ 后的溶液进行过滤, 得到澄清的碳酸氢 镁溶液. 将得到的碳酸氢镁溶液置于 $250 \mathrm{~mL}$ 烧杯中, 在水浴中加热至不同温度 $\left(25^{\circ} \mathrm{C}, 80^{\circ} \mathrm{C}\right)$, 恒温摚拌处 理一段时间, 直至大量固体析出. 将得到的产物过 滤, 多次冲洗, 干燥 $\left(60^{\circ} \mathrm{C}\right)$, 得到 $\mathrm{CO}_{2}$ 矿化产物.

\section{3 矿化产物的表征分析}

实验过程中得到的固相产物通过 X 射线衍射仪 (XRD) 测定其晶体性质. 样品形貌通过扫描电子显 微镜(SEM)进行研究. 产物在空气中的热稳定性通过 热重分析仪(TG)进行测定，将 10 20 mg 样品置于热 重分析仪的样品杯中, 在空气气氛下以 $20^{\circ} \mathrm{C} / \mathrm{min}$ 的 升温速率, 从 $25^{\circ} \mathrm{C}$ 升温至 $800^{\circ} \mathrm{C}$.

\section{2 结果与讨论}

\section{1 电解过程中的电流效率}

电解过程中, 生成产物的实际质量除以由法拉 利定理计算出的理论产量之间的比值称之为电流效 率. 随着电解氯化镁反应的进行, 正极电极液中的盐
酸浓度不断增加. 图 3 显示了不同浓度的盐酸对电流 效率的影响. 当盐酸浓度为 $0.1 \mathrm{~mol} / \mathrm{L}$ 时, 电流效率 可以达到 $98 \%$. 随着盐酸浓度的升高, 电流效率逐渐 降低. 当盐酸浓度为 $1.6 \mathrm{~mol} / \mathrm{L}$ 时, 电流效率下降至 63\%. 造成这一现象的原因是随着氢离子浓度的增加, 阴离子交换膜的选择性降低. 当质子浓度较低时, 阴 离子交换膜阻止了绝大部分氢离子透过，而当氢离 子浓度较高时, 部分质子开始透过阴离子交换膜, 进 人负极电极液, 这使得生成的氢氧化镁减少, 电流效 率下降. 电流效率的下降将导致能耗的增加.

提高阴离子交换膜的选择性，可阻止氢离子的 透过, 从而提高电流效率. 在 Faverjon 等人 ${ }^{[29]}$ 的研究 中, 购买自 Solvay 公司的 ARA 阴离子交换膜表现出 很高的选择性. 当盐酸浓度达到 $4.5 \mathrm{~mol} / \mathrm{L}$ 时, 其电 流效率仍然高达 $75 \%$. 氢离子的透过直接影响了能 够产出的盐酸浓度, 如何提高阴离子交换膜的选择 性是进一步的研究方向.

\section{2 不同浓度的二氧化碳与氢氧化镁的反应活性}

氢氧化镁在溶液中可与 $\mathrm{CO}_{2}$ 发生如下化学反应, 生成碳酸氢镁:

$$
2 \mathrm{CO}_{2}+\mathrm{Mg}(\mathrm{OH})_{2} \rightarrow \mathrm{Mg}\left(\mathrm{HCO}_{3}\right)_{2}
$$

根据 Jung 等人 ${ }^{[31]}$ 的研究结果, 利用氢氧化镁吸 收烟气中的 $\mathrm{CO}_{2}$ 是可行的, 并且这一过程具有较快 的吸收速率. 在该实验过程中, 不同浓度的 $\mathrm{CO}_{2}(20 \%$, $50 \%, 70 \%, 100 \%)$ 被作为反应原料与电解产生的氢氧 化镁进行反应, 溶液中碳酸氢根的浓度随时间的变 化如图 4 所示. 从图 4 中可以看出, 浓度为 $20 \%$ $100 \%$ 的 $\mathrm{CO}_{2}$ 都能直接在常温下与氢氧化镁发生矿化 反应, 因此, 采用该方法可直接矿化浓度仅为 $20 \%$ 的

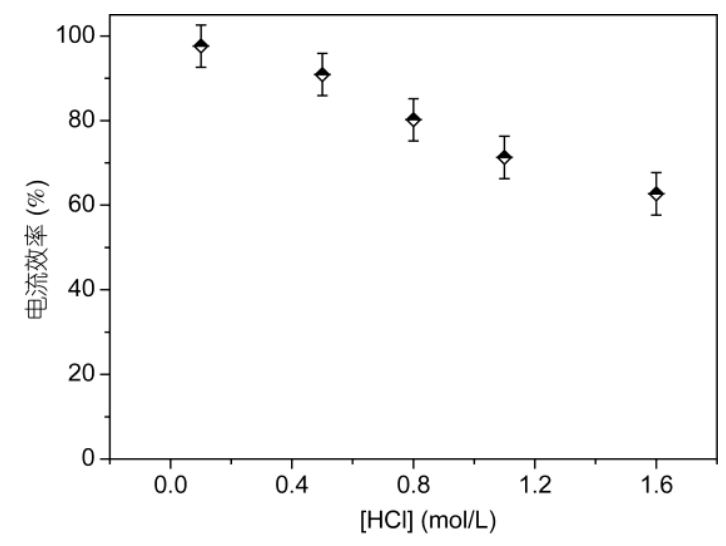

图 3 不同浓度的盐酸对电流效率的影响 
低浓度 $\mathrm{CO}_{2}, \mathrm{CO}_{2}$ 浓度越高, $\mathrm{CO}_{2}$ 的矿化速率越快, 碳酸 氢镁溶液的平衡浓度也越高. 另外, 采用 $100 \% \mathrm{CO}_{2}$ 作 为原料时, 溶液中的碳酸氢根浓度在 $100 \mathrm{~min}$ 后稳定在 $0.4 \mathrm{~mol} / \mathrm{L}$ 左右, 而当采用 $20 \% \mathrm{CO}_{2}$ 作为反应原料时, 反应 $400 \mathrm{~min}$ 后, 溶液中的碳酸氢根浓度为 $0.2 \mathrm{~mol} / \mathrm{L}$.

该研究证实了利用电解反应制取的氢氧化镁具 有较高的反应活性, 能够直接矿化低浓度 $\mathrm{CO}_{2}$. 另外, 除了能够矿化 $\mathrm{CO}_{2}$, 利用氢氧化镁悬浮液进行烟气脱 硫也已被广泛研究, 并取得了很好的效果 ${ }^{[32]}$. 由于 氢氧化镁脱硫后的产物大部分为可溶性的硫酸镁, 并不会影响固体产物碳酸镁的纯度. 因此, 利用该方 法产生的氢氧化镁作为原料, 具有直接矿化烟气 $\mathrm{CO}_{2}$, 避免高能耗的 $\mathrm{CO}_{2}$ 捕捉过程，同时脱除烟气中二氧 化硫的可能.

从图 4 可以看出, 在不同 $\mathrm{CO}_{2}$ 浓度下, 溶液中碳 酸氢根的浓度在反应过程中均呈现先少量升高后下 降, 然后再次明显升高最后趋于稳定的变化趋势. 这 是由于固体氢氧化镁在水中的溶解度较低, 因此, 固体氢氧化镁周围的镁离子含量较高, 当溶液中 的碳酸氢根达到一定浓度后, 便与氢氧化镁周围的 镁离子反应，生成碳酸镁固体，导致反应一定时间 后, 溶液中的碳酸氢根的浓度下降. XRD 研究结果表 明，在碳酸氢根浓度降低时，固体中确实生成了 $\mathrm{MgCO}_{3} \cdot 3 \mathrm{H}_{2} \mathrm{O}$ 晶体(图 5). 由于碳酸镁以及未反应的 氢氧化镁都能与 $\mathrm{CO}_{2}$ 反应, 重新生成碳酸氢镁, 使得 溶液中的碳酸氢根的含量继续升高, 直至溶液中碳 酸氢根的浓度达到饱和.

\section{3 固体产物成分及其热稳定性}

碳酸氢镁溶液在不同温度下分解, 得到碳酸镁

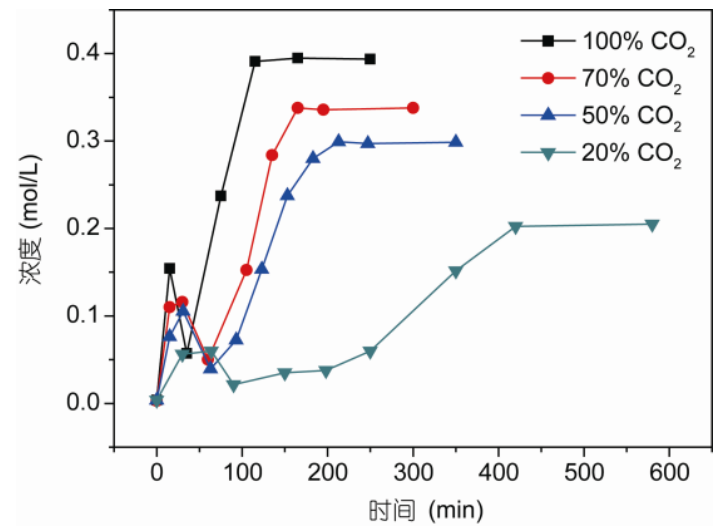

图 4 碳酸氢根的浓度随时间的变化

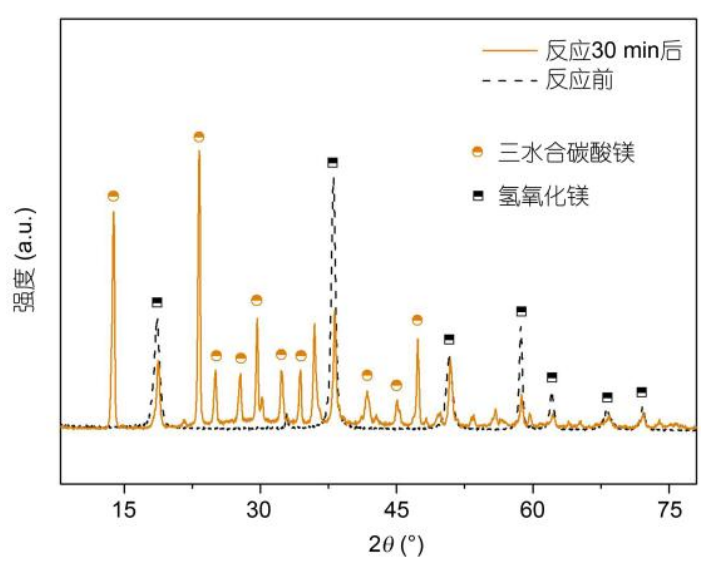

图 5 固相 XRD 分析结果对比

的种类有所不同. 图 6 显示了碳酸氢镁溶液在 $25^{\circ} \mathrm{C}$ 和 $80^{\circ} \mathrm{C}$ 下分解所得产物的 XRD 分析结果. 在 $25^{\circ} \mathrm{C}$ 的 处理温度下搅拌, 碳酸氢镁溶液转变为三水合碳酸 镁 $\left(\mathrm{MgCO}_{3} \cdot 3 \mathrm{H}_{2} \mathrm{O}\right)$, 而在 $80^{\circ} \mathrm{C}$ 的分解温度下, 碳酸氢 镁溶液将转变为碱式碳酸镁 $\left(\mathrm{Mg}_{5}\left(\mathrm{CO}_{3}\right)_{4}(\mathrm{OH})_{2} \cdot 4 \mathrm{H}_{2} \mathrm{O}\right)$. 从产物的 SEM 图可以看出, 三水合碳酸镁为长条形 的长方体结构(图 7(a)), 而碱式碳酸镁呈现出花瓣型 结构(图 7(b)).

产物热重分析结果表明, 常温分解后获得的三水 合碳酸镁在 $100 \sim 300^{\circ} \mathrm{C}$ 和 $300 \sim 550^{\circ} \mathrm{C}$ 的范围内有两 个明显的失重过程(图 8). 这一结果与 Rao 等人 ${ }^{[33]}$ 的报 道一致, $\mathrm{MgCO}_{3} \cdot 3 \mathrm{H}_{2} \mathrm{O}$ 在 $100 \sim 330^{\circ} \mathrm{C}$ 范围内的失去结 晶水生成 $\mathrm{MgCO}_{3}$, 而在 $350 \sim 550^{\circ} \mathrm{C}$ 的范围内, $\mathrm{MgCO}_{3}$ 会分解出 $\mathrm{CO}_{2}$, 生成 $\mathrm{MgO}$. 这进一步印证了碳酸氢镁 溶液在常温下的分解产物为 $\mathrm{MgCO}_{3} \cdot 3 \mathrm{H}_{2} \mathrm{O}$, 并且

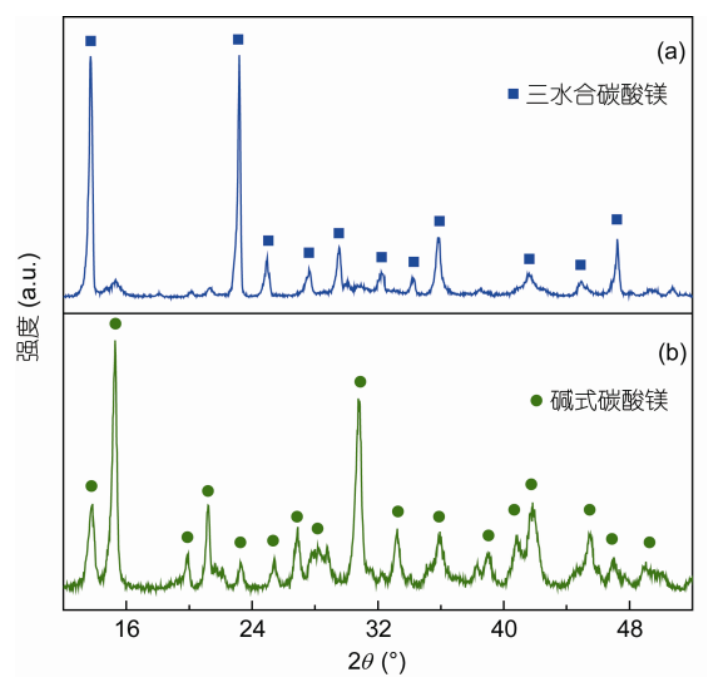

图 6 分解产物的 XRD 分析结果 


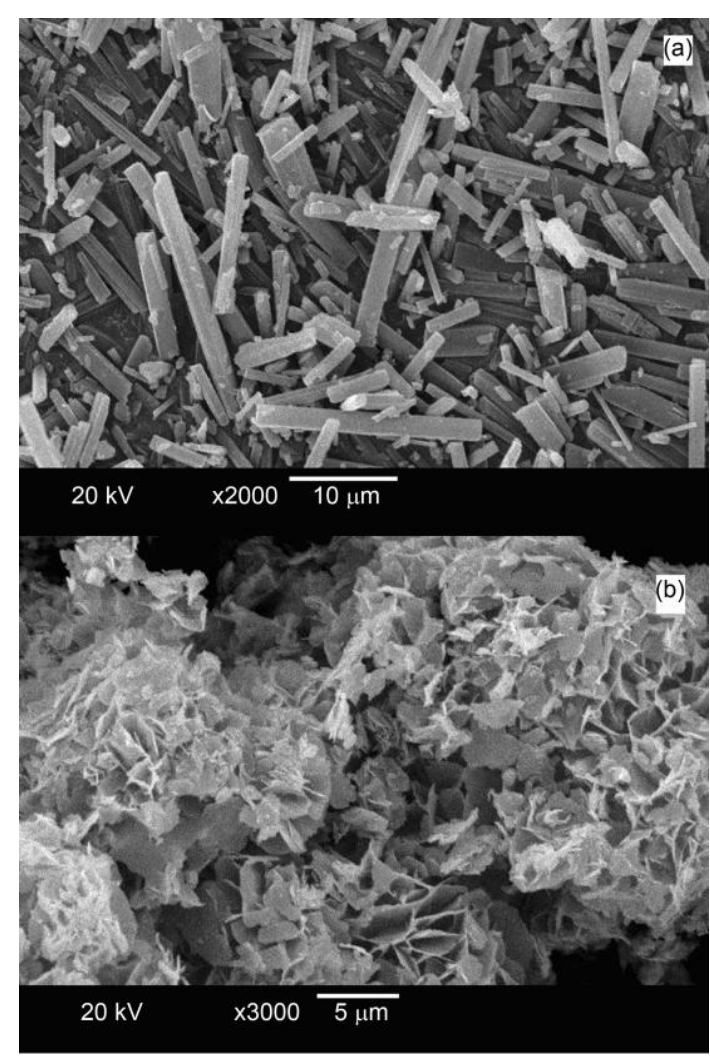

图 7 碳酸氢镁分解产物的 SEM 分析结果

(a) 碳酸氢镁溶液常温分解所得产物; (b) 碳酸氢镁溶液在 $80^{\circ} \mathrm{C}$ 下分解所得产物

$\mathrm{MgCO}_{3} \cdot 3 \mathrm{H}_{2} \mathrm{O}$ 在空气中常温下具有良好的稳定性.

从图 8 可以看出, 碳酸氢镁溶液在 $80^{\circ} \mathrm{C}$ 下分解后 所得产物碱式碳酸镁在 $200 \sim 280^{\circ} \mathrm{C}, 400 \sim 500^{\circ} \mathrm{C}$, $500 \sim 560^{\circ} \mathrm{C} 3$ 个阶段具有明显的失重过程, 这与碱式 碳酸镁的失重曲线基本一致，其中前两个失重阶段 是由于失去结晶水和氢氧化物中的水分子而引起的,

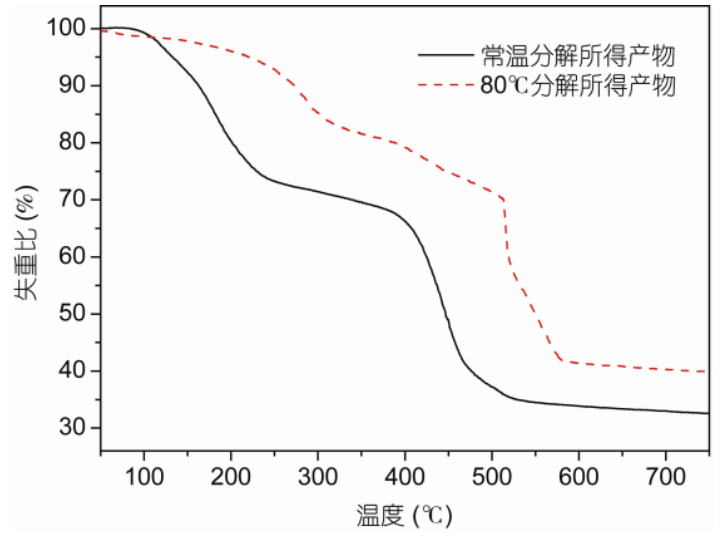

图 8 产物热重分析结果
而大于 $500^{\circ} \mathrm{C}$ 的失重是由于释放 $\mathrm{CO}_{2}$ 所引起的 ${ }^{[34]}$. 这 说明, 碱式碳酸镁在空气中常温下同样具有良好的 稳定性.

\section{4 槽电压及电解能耗}

图 9(a) 显示了采用不同浓度的盐酸作为正极电 极液时, 电流密度随电压变化. 从图 9(a)中可以看出, 当槽电压为 $0.60 \sim 0.62 \mathrm{~V}$ 时, 该反应便开始进行. 随着 盐酸浓度的增加, 电流密度随电压的增加更加明显. 这是因为, 随着盐酸浓度的增加, 正极电极液的离子 强度增加, 电流随电压的变化更为明显. 图 9(b)显示 了采用不同浓度的氯化钠, 氯化镁混合溶液作为负极 电极液时，电流密度随电压变化情况. 从图 9(b)中可 以看出、氯化镁，氯化钠的配比对电流密度随电压的 变化几乎没有影响.

采用氯化镁电化学矿化利用 $\mathrm{CO}_{2}$ 的主要能耗为 氯化镁的电解过程. 电解过程的能耗与槽电压和电 流效率有关. 理论上, 电解氯化镁的最小槽电压仅为
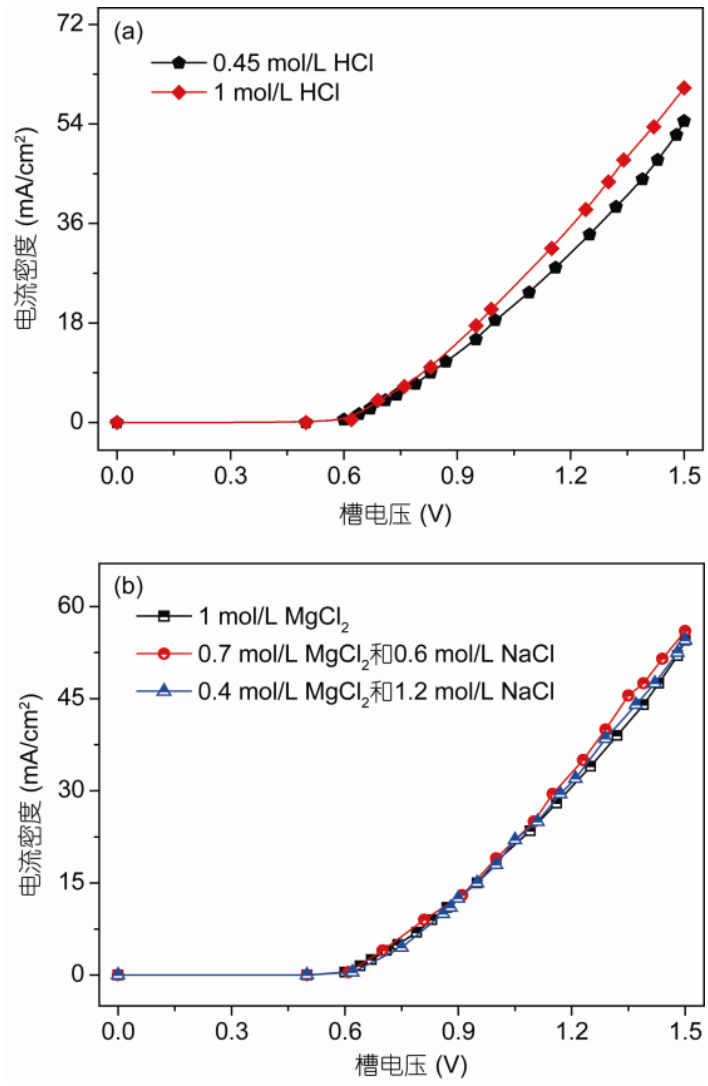

图 9 电流密度随槽电压的变化

(a) 不同浓度的盐酸作为正极电极液; (b) 不同浓度的氯化镁和氯 化钠混合溶液作为负极电极液 
$0.38 \mathrm{~V}$, 在该电压下, 矿化每吨 $\mathrm{CO}_{2}$ 的最小能耗为 $473 \mathrm{~kW} \mathrm{~h}$. 研究表明, 在本体系的实验条件下, 电解 氯化镁的反应在 $0.60 \sim 0.62 \mathrm{~V}$ 时反应便可进行, 但在 该电压下电流太小, 反应速率较低. 提高槽电压可提 高反应速率, 但电解的能耗也随之增加. 当槽电压为 $0.7 \mathrm{~V}$, 盐酸浓度为 $0.1 \mathrm{~mol} / \mathrm{L}$ 时(此时的电流效率为 $98 \%$ ), 矿化每吨 $\mathrm{CO}_{2}$, 联产 3.16 吨碳酸镁(三水合碳 酸镁)的所需的电解能耗约为 $871 \mathrm{~kW} \mathrm{~h}$. 按照每度电 的价格为 0.5 元进行计算, 矿化每吨 $\mathrm{CO}_{2}$, 联产 3.16 吨三水合碳酸镁的电能成本为 435.5 元. 由于获得的 三水合碳酸镁市场价格为 3000 元左右, 因此, 矿化 每吨 $\mathrm{CO}_{2}$ 可获得 9480 元的矿化产物. 因此, 利用氯 化镁溶液矿化利用 $\mathrm{CO}_{2}$ 具有很好的工业应用前景.

\section{3 结论}

氯化镁是地球上分布广泛，来源丰富的化合物.
本文报道了一种利用氯化镁溶液电化学矿化利用 $\mathrm{CO}_{2}$ 的新方法. 该方法首先采用电解工艺将氯化镁转 化为反应活性较高的氢氧化镁, 再通过氢氧化镁将 $\mathrm{CO}_{2}$ 转化为碳酸氢镁溶液, 最后控制碳酸氢镁的分解 温度, 便可将 $\mathrm{CO}_{2}$ 最终矿化为具有高附加值的三水 合碳酸镁或碱式碳酸镁.

本文提出的 $\mathrm{CO}_{2}$ 矿化利用新方法可直接采用浓 度仅为 $20 \%$ 的低浓度 $\mathrm{CO}_{2}$ 作为原料, 这使得直接矿 化利用工业烟气, 避免高能耗的 $\mathrm{CO}_{2}$ 捕捉过程成为 可能, 大幅度降低了 $\mathrm{CO}_{2}$ 的矿化利用成本.

采用氯化镁矿化利用 $\mathrm{CO}_{2}$ 的主要能耗为氯化镁 的电解过程. 电解氯化镁的反应在 $0.60 \sim 0.62 \mathrm{~V}$ 时, 反应便可进行. 为了提高反应速率可适当增加槽电 压, 当槽电压为 $0.7 \mathrm{~V}$, 电流效率为 $98 \%$ 时, 矿化每 吨 $\mathrm{CO}_{2}$, 联产 3.16 吨碳酸镁 (三水合碳酸镁)所需的能 耗约为 $871 \mathrm{~kW} \mathrm{~h}$ (约 435.5 元).

\section{参考文献}

1 Keith D W. Why capture $\mathrm{CO}_{2}$ from the atmosphere? Science, 2009, 325: 1654-1655

2 谢和平. $\mathrm{CO}_{2}$ 封存与气候变化. 科技导报, 2010, 28: 3

3 谢和平. 发展低碳技术、推进绿色经济. 中国能源, 2010, 32: 5-10

4 Karl T R, Trenberth K E. Modern global climate change. Science, 2003, 302: 1719-1723

5 Friedlingstein P, Solomon S, Plattner G K, et al. Long-term climate implications of twenty-first century options for carbon dioxide emission mitigation. Nat Clim Change, 2011, 1: 457-461

6 Lackner K S. A guide to $\mathrm{CO}_{2}$ sequestration. Science, 2003, 300: 1677-1678

7 IPCC. Special Report on Carbon Dioxide Capture and Storage. Cambridge: Cambridge University Press, 2005

8 Jun Y S, Giammar D E, Werth C J. Impacts of geochemical reactions on geologic carbon sequestration. Environ Sci Technol, 2013, 47: 3-8

9 Hou M Z, Xie H P, Yoon J S. Underground Storage of $\mathrm{CO}_{2}$ and Energy. Boca Raton: CRC Press, 2010

10 Haszeldine R S. Carbon capture and storage: How green can black be? Science, 2009, 325: 1647-1652

11 谢和平. $\mathrm{CO}_{2}$ 矿化利用的 $\mathrm{CCU}$ 新理念. 技术报告, 中国工程院能源与矿业学部, 2012

12 谢和平, 谢凌志, 王昱飞, 等. 全球二氧化碳减排不应是 CCS, 应是 CCU. 四川大学学报(工程科学版), 2012, 44: 1-5

13 谢和平, 王昱飞, 鞠杨, 等. 地球自然钾长石矿化 $\mathrm{CO}_{2}$ 联产可溶性钾盐. 科学通报, 2012, 57: 2501-2506

14 Seifritz W. $\mathrm{CO}_{2}$ disposal by means of silicates. Nature, 1990, 345: 486

15 Gerdemann S J, O’Conner W K, Dahlin D C, et al. Ex situ aqueous mineral carbonation. Environ Sci Technol, 2007, 41: 2587-2593

16 Kelemen P B, Matter J. In situ carbonation of peridotite for $\mathrm{CO}_{2}$ storage. Proc Natl Acad Sci USA, 2008, 105: 17295-17300

17 Koukouzas N, Gemeni V, Ziock H J. Sequestration of $\mathrm{CO}_{2}$ in magnesium silicates, in Western Macedonia, Greece. Int J Miner Process, 2009, 93: 179-186

18 Loring J S, Thompson C J, Wang Z, et al. In situ infrared spectroscopic study of forsterite carbonation in wet supercritical $\mathrm{CO}_{2}$. Environ Sci Technol, 2011, 45: 6204-6210

19 Park A H A, Fan L S. $\mathrm{CO}_{2}$ mineral sequestration: Physically activated dissolution of serpentine and pH swing process. Chem Eng Sci, 2004, 59: 5241-5247

20 Fagerlund J, Teir S, Nduagu E, et al. Carbonation of magnesium silicate mineral using a pressurised gas/solid process. Energy Proced, 2009, 1: 4907-4914 
21 Maroto-Valer M M, Fauth D J, Kuchta M E, et al. Activation of magnesium rich minerals as carbonation feedstock materials for $\mathrm{CO}_{2}$ sequestration. Fuel Process Technol, 2005, 86: 1627-1645

22 Huijgen W J J, Comans R N J, Witkamp G J. Cost evaluation of $\mathrm{CO}_{2}$ sequestration by aqueous mineral carbonation. Energ Convers Manage, 2007, 48: 1923-1935

23 Huntzinger D N, Gierke J S, Kawatra S K, et al. Carbon dioxide sequestration in cement kiln dust through mineral carbonation. Environ Sci Technol, 2009, 43: 1986-1992

24 Sun J, Bertos M F, Simons S J R. Kinetic study of accelerated carbonation of municipal solid waste incinerator air pollution control residues for sequestration of flue gas $\mathrm{CO}_{2}$. Energy Environ Sci, 2008, 1: 370-377

25 Huijgen W J J, Witkamp G J, Comans R N J. Mineral $\mathrm{CO}_{2}$ sequestration by steel slag carbonation. Environ Sci Technol, 2005, 39: 9676-9682

26 Stolaroff J K, Lowry G V, Keith D W. Using CaO- and MgO-rich industrial waste streams for carbon sequestration. Energ Convers Manage, 2005, 46: 687-699

27 IEA. $\mathrm{CO}_{2}$ Emission from Fuel Combustion Highlights. France: OECD/IEA, 2011

28 Faverjon F, Durand G, Rakib M. Regeneration of hydrochloric acid and sodium hydroxide from purified sodium chloride by membra ne electrolysis using a hydrogen diffusion anode-membrane assembly. J Membr Sci, 2006, 284: 323-330

29 Faverjon F, Rakib M, Durand G. Electrochemical study of a hydrogen diffusion anode-membrane assembly for membrane electrolysis. Electrochim Acta, 2005, 51: 386-394

30 Holze S, Jorissen J, Fischer C, et al. Hydrogen consuming anodes for energy saving in sodium sulphate electrolysis. Chem Eng Technol, 1994, 17: 382-389

31 Jung K S, Keener T C, Green V C, et al. $\mathrm{CO}_{2}$ absorption study in a bubble column reactor with $\mathrm{Mg}(\mathrm{OH})_{2}$. Int J Environ Technol Manage, 2004, 4: 116-136

32 曹霞, 陈秀萍. 浅谈氢氧化镁脱硫技术. 有色冶金设计与研究, 2000, 21: 47-51

33 Rao T R, Chohan V S. Kinetics of thermal decomposition of hydromagnesite. Chem Eng Technol, 1995, 18: 359-363

34 Hollingbery L A, Hull T R. The thermal decomposition of huntite and hydromagnesite-A review. Thermochim Acta, 2010, 509: 1-11 